\title{
Sistema Inteligente para auxílio na Avaliação e Intervenção de Doenças de Demência
}

\author{
${ }^{1}$ Douglas Dotto, ${ }^{1,3}$ Rejane Frozza, ${ }^{2}$ Daielle Marion, ${ }^{2}$ Silvia Virginia Coutinho Areosa, \\ ${ }^{2,3}$ Liane Mahlmann Kipper
}

\author{
Universidade de Santa Cruz do Sul (UNISC) Av. Independência, 2293 - Bairro \\ Universitário Santa Cruz do Sul - RS - Brasil \\ ${ }^{1}$ Departamento de Engenharias, Arquitetura e Computação \\ ${ }^{2}$ Mestrado Profissional em Psicologia \\ ${ }^{3}$ Programa de Pós-graduação em Sistemas e Processos Industriais \\ ddotto@mx2.unisc.br, frozza@unisc.br, daiellemarion@yahoo.com.br, \\ sareosadunisc.br, lianedunisc.br
}

\begin{abstract}
Resumo. Com o processo de rápido envelhecimento da população brasileira, é necessário repensar a forma de atendimento dos usuários idosos que utilizam o Sistema Único de Saúde (SUS), para que seja possível auxiliar na melhoria de qualidade do atendimento destes usuários. Esta pesquisa tem foco nas doenças de demência, especificamente na sua avaliação e intervenção, e também na área da computação. O objetivo é auxiliar na identificação de doenças de demência, a partir da combinação de sintomas existentes, assim como no resultado de testes padrão utilizados por profissionais da saúde. A pesquisa está em andamento e o sistema será desenvolvido para dispositivo móvel, a fim de facilitar o acesso por profissionais da área.
\end{abstract}

Abstract. With the process of rapid aging of the Brazilian population, it is necessary to rethink the form of care provided to elderly users using the Unified Health System (SUS), so that it is possible to assist in improving the quality of care of these users' lives. This research focuses on dementia diseases, specifically in their assessment and intervention, and also in the area of computing. The objective is to assist in the identification of dementia diseases, based on the combination of existing symptoms, as well as the result of standard tests used by health professionals. The research is ongoing and the system will be developed for mobile devices and also on a web platform, in order to facilitate access by professionals in the field.

\section{Introdução}

O Brasil atravessa, atualmente, um processo de rápido envelhecimento da população. De acordo com o Instituto Brasileiro de Geografia e Estatística (IBGE), já são 30,2 milhões de idosos. É estimado que esse contingente triplique nos próximos 30 anos. O número de idosos no Brasil vai ultrapassar o número de jovens em 2031, com 42,3 milhões de jovens entre 0 a 14 anos e 43,3 milhões de idosos com 60 anos ou mais. Em 2031 este índice será maior do que 100, ou seja, haverá 102,3 idosos para cada 100 jovens. O Rio Grande do Sul deve ser o primeiro estado a ultrapassar este índice, em meados de 2030 (IBGE, 2018).

Como os idosos têm maior propensão às doenças de demência, a prioridade do sistema inteligente é a identificação das doenças de demência existentes para auxiliar na tomada de decisão e consequentemente contribuir para uma reabilitação precoce, a fim de 
diminuir o impacto das condições crônicas, buscando a intervenção antes da ocorrência de um agravo. A ideia é monitorar a saúde do idoso, a fim de que ele possa usufruir com qualidade seu tempo de vida (Veras et al., 2013).

Assim, este trabalho pretende responder o seguinte problema de pesquisa: É possível auxiliar os profissionais de saúde, que prestam serviços aos idosos no SUS, no diagnóstico de doenças de demência por meio de análise histórica e ainda auxiliar na tomada de decisão dos médicos para uma melhora dos sintomas clínicos ou até mesmo no retardo ao avanço da demência em determinados indivíduos?

No intuito de adquirir esta resposta, será desenvolvido um sistema inteligente (sistema de apoio à decisão baseado em conhecimento) com objetivo de prestar auxílio na tomada de decisão, devendo reunir as principais informações dos idosos (histórico de testes aplicados, histórico de uso de medicações, intercorrências, sinais vitais e histórico clínico) e, então, apresentar como resultado uma possível indicação de diagnóstico de doença de demência.

O artigo está organizado nas seguintes seções: a seção 2 apresenta uma breve fundamentação teórica, a seção 3 descreve a metodologia, na seção 4 são abordados os aspectos referentes à proposta do trabalho e a seção 5 apresenta as considerações finais.

\section{Fundamentação teórica}

As doenças de demência são caracterizadas pelo declínio cognitivo ou modificações comportamentais (neuropsiquiátricas) em relação a um nível prévio de desempenho, causando a perda da independência para as atividades diárias. A identificação do declínio cognitivo visa intervir precocemente em causas secundárias e reversíveis, bem como planejar o cuidado dos pacientes com demência (Regula SUS, 2016).

Os tipos de demência mais comuns e que serão analisadas por este sistema inteligente são: Doença de Alzheimer (DA); Demência Vascular (DV); Demência de corpúsculos de Lewy; Demências Frontotemporais e Doença de Pick (Vigêta, 2011).

Já a Atenção Básica caracteriza-se por um conjunto de ações de saúde, no âmbito individual e coletivo, que abrange a promoção e a proteção da saúde, a prevenção de agravos, o diagnóstico, o tratamento, a reabilitação e a manutenção da saúde. É desenvolvida por meio do exercício de práticas gerenciais e sanitárias democráticas e participativas, sob forma de trabalho em equipe, dirigidas a populações de territórios bem delimitados, pelas quais assume a responsabilidade sanitária, considerando a dinamicidade existente no território em que vivem essas populações. Utiliza tecnologias de elevada complexidade e baixa densidade, que devem resolver os problemas de saúde de maior frequência e relevância em seu território. É o contato preferencial dos usuários com os sistemas de saúde. Orienta-se pelos princípios da universalidade, da acessibilidade e da coordenação do cuidado, do vínculo, da continuidade, da integralidade, da responsabilização, da humanização, da equidade e da participação social (BRASIL, 2017).

Segundo Rezende (2003), um sistema inteligente, baseado em conhecimento, apresenta um conjunto de regras e sentenças que possibilitam mecanismos de raciocínio e conhecimento capaz de inferir melhores soluções aos problemas propostos.

Os pontos chaves nos sistemas inteligentes são: i) habilidades para usar conhecimento para desempenhar tarefas ou resolver problemas e; ii) a capacidade de trabalhar com problemas complexos em aplicações reais e que podem ser desenvolvidas com o uso de 
algumas técnicas (Rezende, 2003). Dessa forma, capturando o conhecimento de um especialista, organizando-o e disponibilizando-o em conjunto com o histórico dos testes aplicados e demais informações coletadas pela Equipe de Estratégia Saúde de Família (ESF), é possível indicar para o médico responsável qual a probabilidade de determinado paciente possuir uma ou mais doenças de demência descritas anteriormente. $\mathrm{O}$ intuito é auxiliar no processo de tomada de decisão.

\section{Materiais e Métodos}

Uma bibliometria quantitativa (Araújo, 2006) foi realizada, com o objetivo de verificar as produções científicas, que abordam o tema de pesquisa deste trabalho, no período de janeiro de 2015 a janeiro de 2020. Para isto, foram utilizados os termos de busca "dementia disease", "health intervention" e "intelligent systems". As bases de dados escolhidas foram SciELO, Scopus e Pubmed, utilizando Computer Science como área de pesquisa, e filtrando apenas por artigos científicos. Foram encontrados 188 trabalhos com os termos de busca em conjunto. Então, foram selecionados quatro artigos, após leitura dos resumos para serem estudados e contribuírem para o desenvolvimento da pesquisa, sendo ele: Urwyler et al. (2017), Livingston et al. (2017), Lorenz et al. (2017) e Tyack et al. (2017). Todos estes trabalhos citam a utilidade do uso de ferramentas e tecnologias para pacientes com doenças de demência, com coleta de dados e auxílio ao processo de tomada de decisão, propondo uma estabilização do comprometimento cognitivo, do comportamento e da realização das atividades da vida diária (ou modificar as manifestações da doença), com o mínimo de efeitos adversos e melhorando o bem-estar geral.

Os procedimentos metodológicos definidos são: i) levantamento bibliográfico, com estudos realizados para obter aprofundamento nos temas da pesquisa; ii) levantamento de trabalhos relacionados, elaborando sínteses e quadro comparativo; iii) entrevista com especialista humano para iniciar a modelagem do sistema baseado em conhecimento para auxiliar no diagnóstico de doenças de demência e na intervenção; iv) processo de representação do conhecimento, a elaboração das regras para a base de conhecimento do sistema, juntamente com especialista humano; v) desenvolvimento do aplicativo utilizando as técnicas que envolvem a detecção de doenças de demência e vi) validação do processo com especialista humano e usuários.

\section{Proposta: Sistema inteligente para auxílio na avaliação e intervenção de doenças de demência}

O usuário interagirá com o sistema inteligente (sistema especialista) por meio de um aplicativo em um dispositivo móvel, conforme ilustra a Figura 1. Na forma de Web Service, o dispositivo realizará os testes e enviará ao servidor, que executará o processamento dos dados. Para isso, utilizará a base de conhecimento, gerada com o conhecimento do especialista humano, modelado em árvore de decisão, sendo manipulado pelo motor de inferência, para, então, retornar os resultados para a aplicação do dispositivo móvel. Após, serão exibidos os resultados dos testes e possíveis diagnósticos de doenças de demência, auxiliando na avaliação e intervenção. 


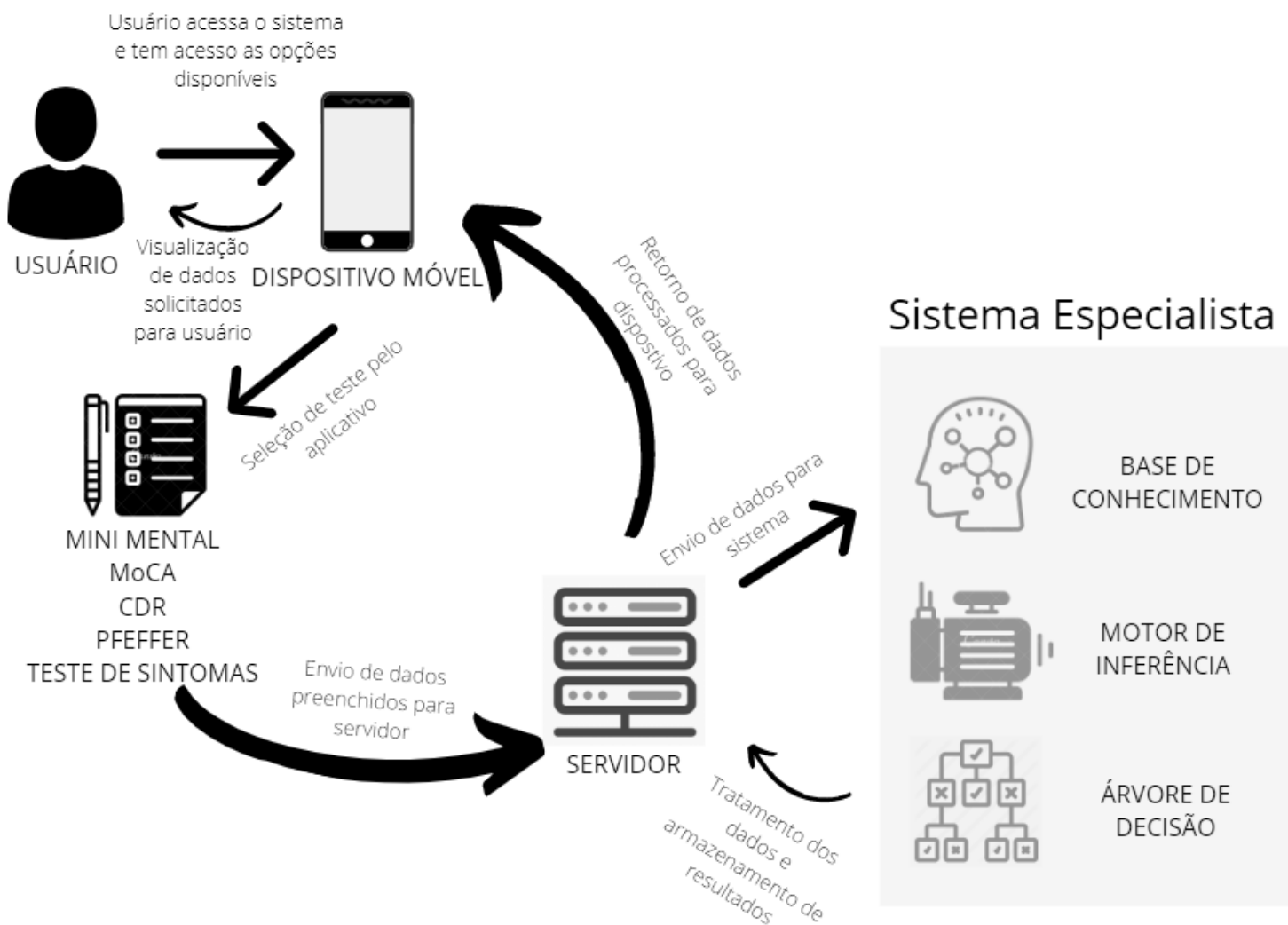

Figura 1 - Sistema Proposto

Os métodos a seguir, ilustrados na Figura 2, foram organizados em conjunto com as especialistas humanas da área do Mestrado Profissional em Psicologia da UNISC.

As demandas de saúde da população chegam ao conhecimento da Equipe de Estratégia Saúde de Família (ESF) de duas maneiras: i) Através do contato com os Agentes Comunitários de Saúde (ACS) que, posteriormente, levam os casos a sua coordenação (médico ou enfermeira ESF); ii) Através da procura direta por atendimento na Unidade Básica de Saúde (UBS) pelo paciente ou familiar responsável, que é atendido por um técnico de enfermagem que acolhe sua queixa.

O $1^{\circ}$ passo é a aplicação do Questionário Pfeffer, pelo agente comunitário de saúde ou pelo profissional que for realizar a visita domiciliar. Este teste avalia o desempenho em dez atividades instrumentais de vida diária (AIVD) que envolvem também habilidades cognitivas: controlar as próprias finanças, fazer compras, esquentar água e apagar o fogo, preparar refeições, manter-se atualizado, prestar atenção em uma notícia e discuti-la, lembrar-se de compromissos, cuidar da própria medicação, manter-se orientado ao andar pela vizinhança e ficar sozinho em casa (Assis et al., 2015).

O $2^{\circ}$ passo é a Avaliação Clínica da Demência (CDR), sendo esse teste aplicado pela equipe de enfermagem que faz o acolhimento e triagem do paciente. A CDR é uma entrevista semiestruturada, respondida pelo paciente e por um informante, que avalia o nível de funcionamento cognitivo e de atividades diárias atuais em comparação às habilidades prévias do paciente. A CDR abrange seis domínios: memória, orientação, julgamento e solução de problemas, assuntos da comunidade, lar e passatempos e cuidados pessoais (Lima, 2016). 


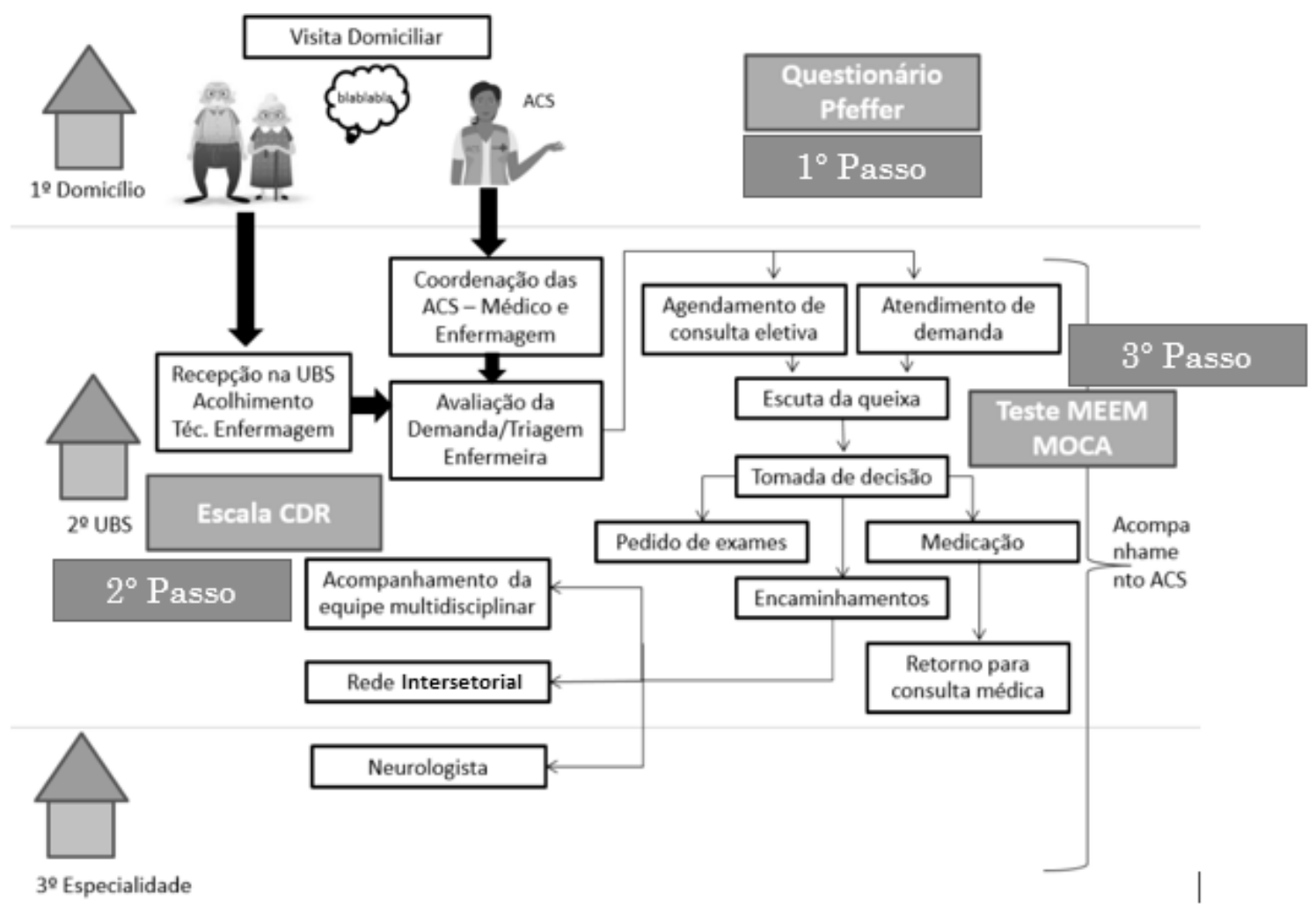

Figura 2 - Fluxo de Atendimento

No $3^{\circ}$ passo, diante da percepção de queixas relacionadas à memória ou até mesmo comportamento, o médico ou enfermeira pode aplicar o Mini Exame do Estado Mental (MEEM), disponível através do Projeto Telessaúde RS ou o teste MoCA. A partir disso ocorre a tomada de decisões, dentre as quais é possível citar: i) A solicitação de exames complementares; ii) A prescrição de medicação; iii) $\mathrm{O}$ encaminhamento do paciente.

\section{Considerações Finais}

A pesquisa em andamento propõe desenvolver um sistema baseado em conhecimento para auxílio aos profissionais de Saúde das ESFs de Lajeado - Rio Grande do Sul e Colinas - Rio Grande do Sul na avaliação e intervenção de pacientes com doença de demência. A técnica de Entrevistas será utilizada no processo de aquisição do conhecimento junto aos especialistas humanos da área da Psicologia. A representação do conhecimento do domínio será realizada por meio de árvores de decisão e regras, a fim de auxiliar na tomada de decisões dos médicos em relação a doenças de demência. O sistema será desenvolvido para aplicativo móvel e um painel web, a fim de facilitar o trabalho dos profissionais da área. A avaliação do sistema inteligente será realizada na ESF citada, juntamente com a profissional, especialista humana, atuante nesta pesquisa.

\section{Referências}

ARAÚJO, C. A. Bibliometria: evolução histórica e questões atuais. Em Questão, Porto Alegre, v. 12, n. 1, p. 11-32, 2006.

ASSIS, L. O. et al. O questionário de atividades de Pfeffer: revisão integrativa da literatura brasileira. Estudos interdisciplinares sobre o Envelhecimento. Porto Alegre, v. 20, n. 1, p. 297-324, 2015. 
BRASIL. Ministério da Saúde. Plano de cuidado para idosos na saúde suplementar. Distrito Federal: Ministério da Saúde, 2012. Disponível em: http://bvsms.saude.gov.br/bvs/publicacoes/plano_cuidado_idosos.pdf. Acesso em: 20 jun. 2019.

BRASIL. Ministério da Saúde. Sistema Nacional de Saúde. Atenção Básica. 2017. Disponível em: https://www.saude.gov.br/artigos/770-sistema-nacional-de-saude/40315atencao-basica>. Acesso em: 23 jun. 2020.

IBGE. Projeção da População (revisão 2018), Rio de Janeiro: 2018. Disponível em: https://www.ibge.gov.br/estatisticas-novoportal/sociais/populacao/9109-projecao-dapopulacao.html. Acesso em: 20 jun. 2019.

LIMA, A. P. V. Avaliação do valor diagnóstico da escala de Avaliação Clínica da Demência (CDR) utilizando o sistema de escore de soma das caixas para detecção de comprometimento cognitivo e demência. Dissertação (Pós-graduação em Medicina) Universidade Federal do Rio Grande do Sul, Porto Alegre, 2016.

REGULA SUS. Resumo Clínico - Demência, 2016. Disponível em: https://www.ufrgs.br/telessauders/documentos/protocolos_resumos/neurologia_resumo_ demencia_TSRS.pdf. Acesso em: 13 maio 2020.

REZENDE, Solange Oliveira (Org.). Sistemas inteligentes: fundamentos e aplicações. Barueri: Manole, 2003.

VERAS, R. P. et al. Desenvolvimento de uma linha de cuidados para o idoso: hierarquização da atenção baseada na capacidade funcional. Rev. bras. geriatr. Gerontol, v. 16, n. 2, p. 385-392, Rio de Janeiro, 2013. Disponível em: http://www.scielo.br/pdf/rbgg/v16n2/18.pdf. Acesso em: 22 jun. 2019.

VIGÊTA, S. M. G. Demência Caso Complexo 6 - Dona Margarida. São Paulo: UNIFESP, $2011 . \quad$ Disponível em: https://www.unasus.unifesp.br/biblioteca_virtual/esf/1/casos_complexos/Dona_Margari da/Complexo_06_Margarida_Demencia.pdf. Acesso em: 13 maio 2020. 